\title{
The association of meat consumption and breast cancer risk: A case control study in a population of Iranian women
}

\author{
Zeinab Karimi $^{1}$, Zahra Bahadoran ${ }^{2}$, Anahita Houshiar-rad ${ }^{3}$, Hamid-Reza Mirzayi ${ }^{4}$, \\ Bahram Rashidkhani ${ }^{5}$ \\ ${ }^{1}$ Faculty of Nutrition Sciences and Food Technology, National Nutrition and Food Technology Research Institute, Shahid Beheshti University \\ of Medical Sciences, Tehran, Iran \\ ${ }^{2}$ Nutrition and Endocrine Research Center, and Obesity Research Center, Research Institute for Endocrine Sciences, Shahid Beheshti \\ University of Medical Sciences, Tehran, Iran \\ ${ }^{3}$ National Nutrition and Food Technology Research Institute, Shahid Beheshti University of Medical Sciences, Tehran, Iran \\ ${ }^{4}$ Department of Radiation Oncology, Faculty of Medicine, Shahid Beheshti University of Medical Sciences, Tehran, Iran \\ ${ }^{5}$ Department of Community Nutrition, National Nutrition and Food Technology Research Institute, Faculty of Nutrition Sciences and Food \\ Technology, Shahid Beheshti University of Medical Sciences, Tehran, Iran
}

\section{Email address: \\ b_rashidkhani@sbmu.ac.ir (B. Rashidkhani)}

\section{To cite this article:}

Zeinab Karimi, Zahra Bahadoran, Anahita Houshiar-rad, Hamid-Reza Mirzayi, Bahram Rashidkhani. The Association of Meat Consumption and Breast Cancer Risk: A Case Control Study in a Population of Iranian Women. American Journal of Life Sciences. Special Issue: Nutrition and Cancer. Vol. 3, No. 2-1, 2015, pp. 6-11. doi: 10.11648/j.ajls.s.2015030201.12

\begin{abstract}
Objective: Epidemiologic data do not provide consistent evidence for an association between consumption of meat and breast cancer risk. We conducted a hospital-based case-control study during April and July 2010 among Iranian women to investigate associations between dietary meat intake, its types and breast cancer risk. Methods: One-hundred consecutively recruited cases with newly diagnosed breast cancer were frequency matched to 175 controls by age. Dietary intake was assessed by using a validated semi-quantitative food frequency questionnaire. Odds ratios and $95 \%$ confidence interval were obtained by using multiple logistic regression models adjusted for various potentially confounding variables. Results: The mean age of participant was $46.2 \pm 8.9$ and $45.9 \pm 9.4 \mathrm{y}$ in cases and controls, respectively. After adjustment of potential confounders, no association was found between total meat intake and the odds of breast cancer, but the risk of breast cancer in the forth quartile of red meat intake, compared with first quartile, significantly increased $(\mathrm{OR}=2.43,95 \% \mathrm{CI}=1.11$ 5.32). Consumption of poultry $>212 \mathrm{~g} /$ week significantly decreased the risk of breast cancer. Higher intake of fish meat decreased the odds of breast cancer ( $\mathrm{P}$ for trend $<0.05$ ), whereas higher intake of processed meat was accompanied with increased the risk of breast cancer $(\mathrm{P}$ for trend $<0.05$ ). Conclusion: We found a positive association between dietary intake of red meat and processed meat products with the odds of breast cancer, as well as protective effects of fish and poultry intake with breast cancer.
\end{abstract}

Keywords: Breast Cancer, Meat, Red Meat, Poultry, Fish, Processed Meat

\section{Introduction}

Breast cancer is the second leading cause of cancer deaths in female today and is the most common cancer among women in both developing and developed countries (1). Annually, more than one million new cases of breast cancer are diagnosed in the worldwide (2). The breast cancer incidence rate among Asian population is estimated intermediate (30-50 per 100,000) (3). Among Iranian women breast cancer is the most common cancer with an incidence rate of 22 per 100,000 (4). Several risk factors including genetics, family history of breast cancer, parity, age at first birth, age at menarche and menopause, breast feeding duration, socio-economic status, oral contraceptives and hormone replacement therapy, body mass index and adulthood weight gain, physical activity and other lifestyle determinants, have been considered for breast cancer (5-7). Among these factors, eating patterns and dietary intakes are 
thought to be important factor and potentially modifiable approach for breast cancer $(8,9)$. Based on previous reports some animal foods such as meat and especially red meat and processed meat products may also impact breast cancer risk through high content of fat, saturated fat, exogenous hormones, and some compounds generated during food processing including heterocyclic amines, $N$-nitroso components and polyaromatic hydrocarbons (10-13). Several prospective and case-control studies have examined the association between meat intake and breast cancer risk but the results have been inconsistent (13-17). In the current case-control study we examined the association between consumption of total meat, red meat, poultry and fish meat, and processed meat products with breast cancer risk among a population of Iranian women.

\section{Subjects and Methods}

\subsection{Study Design and Population}

This hospital-based case-control study was conducted among Iranian women from April and July 2010. Onehundred consecutively recruited cases with primary diagnosis of breast cancer (indentified within 5 month of diagnose) were age-matched to 175 controls. Cases were recruited from women aged 30-65 years, with histological confirmed breast cancer who referred to oncology, radiotherapy, chemotherapy or surgery sectors of Shohada-e-Tajrish hospital. Participants with the history of any type of cancer or cyst (excluding current breast cancer), history of hormone therapy or special diet were excluded from the study. Age-matched controls without any history of cancers or cyst, hormone therapy or special diet, were also recruited from the individuals referred to other sectors of the hospital. Informed written consents were obtained from all participants and the study protocol was approved by the research council of the Research Institute for Nutrition and Food Sciences, Shahid Beheshti University of Medical Sciences.

\subsection{Socio-Demographic, Anthropometrics and Physical Activity}

Data regarding socio-demographic factors including age, educational level, occupation, ethics, life aria, alcohol and tobacco use, medical history of disease, familial history of breast cancer or other cancers, history of hormone therapy, medications and supplements, oral contraceptives, age at menarche, marital status, number of full pregnancies, menopause status and other lifestyle-related factors were collected by trained interviewers. Weight was measured to the nearest $100 \mathrm{~g}$ using digital scales, while the subjects were minimally clothed, without shoes. Height was measured to the nearest $0.5 \mathrm{~cm}$, in a standing position without shoes, using a tape meter. Body mass index was calculated as weight $(\mathrm{kg})$ divided by square of the height $\left(\mathrm{m}^{2}\right)$. Physical activity level was assessed using the validated questionnaire to obtain frequency and time spent on light, moderate, hard and very hard intensity activities according to the list of common activities of daily life over the past year. Physical activity levels were expressed as metabolic equivalent hours per week (METs h/wk).

\subsection{Dietary Assessment}

Dietary data were collected using a validated semiquantitative food frequency questionnaire (FFQ) with 168 food items. This FFQ was developed for dietary assessment of the participants of the Tehran Lipid and Glucose Study (TLGS); the validity and reliability of the FFQ were previously assessed in a random sample, by comparing the data from two FFQs completed $1 \mathrm{y}$ apart and comparing the data from the FFQs and 12 dietary recalls, respectively. Trained dietitians asked participants to designate their intake frequency for each food item consumed during the past year on a daily, weekly, or monthly basis. Portion sizes of consumed foods reported in household measures were then converted to grams. Mean daily intakes of energy and nutrient for each individual were calculated using the Food Composition Table. Total meat intakes were estimated as the sum intake (g/week) of red meat (beef and lamb meat), poultry meat (chicken and other poultry meat), fish meat, and processed meat products including bacon, sausage, salami and hamburger.

\subsection{Statistical Analysis}

All statistical analysis was performed using SPSS (Version 16.0; Chicago, IL). A $P$ value $<0.05$ was used as the statistical evaluation tool. Energy-adjusted meat intake was calculated as [(meat $\times 1000) /$ energy intake], and was assigned as quartiles based on their $25^{\text {th }}-50^{\text {th }}-75^{\text {th }}$ percentile values. Differences in general characteristics between the cases and controls were compared using by the analysis of variance for continuous variables and the chi-square test for categorical variables. The mean of age, weight, BMI, physical activity, energy intake, energy density of diet, and other food groups were determined across quartiles by using the general linear model with adjustment for age, and energy intake.

The odds ratio and $95 \%$ confidence interval of breast cancer in each quartile of meat intake was determined by multivariable logistic regression models with adjustment for potential confounding variables. The following potential confounders were included in the final multivariate logistic regression models: age $(\mathrm{y})$; BMI $(\mathrm{kg} / \mathrm{m} 2)$; educational level (y); occupation (housekeeper/ employee/ retired); use of alcohol and tobacco (yes/no); age at menarche (y); marital status (not married, married, divorced, widow); age at firs pregnancy (y); number of full pregnancy; menopause status (yes/no); family history of breast cancer (yes/no); use of OCP (yes/no); use of bra $(<12 \mathrm{~h},>12 \mathrm{~h})$; life satisfaction (yes/no/partly); physical activity (MET-h/week); energy intake $(\mathrm{kcal} / \mathrm{d})$; energy density of diet ( $\mathrm{kcal} / 100 \mathrm{~g}$ foods). To assess the overall trends of odds ratios across increasing quartiles of meat intake, the median of each quartile was used as a continuous variable in logistic regression models. 


\section{Results}

The mean age of participant was $46.2 \pm 8.9$ and $45.9 \pm 9.4 \mathrm{y}$ in cases and controls, respectively. The mean age at menarche significantly was lower and the mean age at first pregnancy was significantly higher in cases as compared with controls $(\mathrm{P}<0.01)$. The use of tobacco and oral contraceptives were significantly higher, while life satisfaction was significantly lower in women with diagnosed breast cancer as compared with controls $(\mathrm{P}<0.05)$. There were no significant differences in BMI, physical activity, energy intakes, menopause status, educational levels, occupation, marital status and family history of breast cancer between two groups. Women with diagnosed breast cancer compared with controls, significantly consumed more total meat $(596 \pm 365$ vs. $552 \pm 298 \mathrm{~g} /$ week, $\mathrm{P}<0.01)$, red meat $(230 \pm 183$ vs. $158 \pm 123 \mathrm{~g} /$ week, $\mathrm{P}<0.01$ ), and processed meat ( $55 \pm 119$ vs. $46 \pm 84 \mathrm{~g} /$ week, $\mathrm{P}<0.01)$. Dietary intake of poultry and fish meat was statistically similar between the groups. Characteristics of the study participants across quartile categories of energy-adjusted total meat intake are shown in Table 1. There were no significant differences between age, physical activity, weight, BMI and menopausal status across quartile categories. Dietary intakes of energy, energy density, and energy-adjusted intake of total meat and its types, and other food groups across quartile categories are provided in Table 2. Dietary energy intake significantly increased across increasing meat intake ( $\mathrm{P}$ for trend $<0.001$ ), while dietary energy density was constant. Energy-adjusted total meat intake (g/week) in the highest quartile was more than 4 times than lowest quartile category (1010 19 vs. $250 \pm 19 \mathrm{~g} /$ week). Differences between energy-adjusted dietary intake of vegetables, fruits, dairy and legumes were not significant across quartile categories of meat intake. The odds ratio and $95 \%$ CI of breast cancer across quartiles of energy-adjusted meat intake are presented in Table 3. After adjustment for potential confounding variables, no significant association between total meat intake and breast cancer risk was observed whereas higher intake of red meat was accompanied with increased risk of breast cancer ( $\mathrm{P}$ for trend $<0.001)$; the risk of breast cancer in the forth quartile of red meat intake significantly increased $(\mathrm{OR}=2.43,95 \%$ $\mathrm{CI}=1.11-5.32$ ). An inverse association was found between dietary intake of poultry and the risk of breast cancer $(\mathrm{OR}=0.43,95 \% \mathrm{CI}=0.19-0.96$ in the third quartile and $\mathrm{OR}=0.36,95 \% \mathrm{CI}=0.16-0.84$ in the forth quartile). Higher intake of fish meat decreased the odds of breast cancer ( $\mathrm{P}$ for trend $<0.05)$, whereas higher intake of processed meat was accompanied with increased the risk of breast cancer $(\mathrm{P}$ for trend $<0.05$ ).

Table 1. Characteristics of participants across quartile categories of energy-adjusted total meat intake ${ }^{*}$

\begin{tabular}{|c|c|c|c|c|c|}
\hline & \multicolumn{5}{|c|}{ Quartile of total meat intake (g/week) } \\
\hline & $<350$ & $350-496$ & 496-726 & $>726$ & $\boldsymbol{P}^{* *}$ \\
\hline Case/control & $29 / 39$ & $21 / 48$ & $24 / 45$ & $32 / 36$ & 0.61 \\
\hline Number & 68 & 69 & 69 & 68 & \\
\hline Age $(y)$ & $46.1 \pm 9.4$ & $45.8 \pm 9.1$ & $46.5 \pm 7.5$ & $45.8 \pm 10.5$ & 0.91 \\
\hline Physical activity (MET-h/week) ${ }^{\uparrow}$ & $39.5 \pm 0.5$ & $37.6 \pm 0.5$ & $38.6 \pm 0.5$ & $38.6 \pm 0.5$ & 0.09 \\
\hline Weight $(\mathrm{kg})^{\uparrow}$ & $73 \pm 1.6$ & $71.3 \pm 1.7$ & $72.0 \pm 1.7$ & $73.7 \pm 1.8$ & 0.70 \\
\hline Body mass index $\left(\mathrm{kg} / \mathrm{m}^{2}\right)^{\uparrow}$ & $29.2 \pm 0.6$ & $29.6 \pm 0.6$ & $29.0 \pm 0.6$ & $30.0 \pm 0.7$ & 0.72 \\
\hline Age at menarche $(y)$ & $13.6 \pm 1.6$ & $14.0 \pm 1.4$ & $13.8 \pm 1.8$ & $13.3 \pm 1.6$ & 0.07 \\
\hline Age at menopause $(y)$ & $48.8 \pm 4.9$ & $48.9 \pm 5.7$ & $48.1 \pm 3.6$ & $48.2 \pm 4.4$ & 0.91 \\
\hline Age at first birth $(y)$ & $20.9 \pm 4.7$ & $20.9 \pm 4.1$ & $20.5 \pm 4.2$ & $19.6 \pm 3.9$ & 0.38 \\
\hline Family history of breast cancer $(n)$ & 13 & 16 & 10 & 11 & 0.51 \\
\hline
\end{tabular}

${ }^{*}$ Data are mean \pm SD or mean \pm SE unless stated otherwise.

${ }^{* *}$ Analysis of variance or general linear model (with adjustment for age) was used to compare continuous variables and the chi-square test for categorical variables.

${ }^{\top}$ Age-adjusted mean: The mean \pm SD of total meat intake was $257 \pm 101,450 \pm 181,548 \pm 220$, and $818 \pm 384$ in the $1^{\text {th }}, 2^{\text {th }}, 3^{\text {th }}$, and $4^{\text {th }}$, respectively.

Table 2. Dietary intakes of participants across quartile categories of energy-adjusted meat intake*

\begin{tabular}{|c|c|c|c|c|c|}
\hline & \multicolumn{5}{|c|}{ Quartile of total meat intake (g/week) } \\
\hline & $<350$ & $<350$ & $<350$ & $<350$ & $<350$ \\
\hline Energy $(\mathrm{kcal} / \mathrm{d})$ & $2210 \pm 108$ & $2554 \pm 107$ & $2645 \pm 107$ & $3355 \pm 108$ & 0.001 \\
\hline Energy density (kcal/100g of foods) & $84 \pm 2.4$ & $88 \pm 2.4$ & $86 \pm 2.4$ & $87 \pm 2.4$ & 0.60 \\
\hline Total meat ( $g /$ week) & $250 \pm 19$ & $416 \pm 19$ & $597 \pm 19$ & $1010 \pm 19$ & 0.001 \\
\hline Poultry (g/week) & $98 \pm 14$ & $169 \pm 14$ & $212 \pm 14$ & $329 \pm 14$ & 0.001 \\
\hline Red meat ( $g /$ week) & $109 \pm 15$ & $134 \pm 15$ & $182 \pm 15$ & $315 \pm 16$ & 0.001 \\
\hline Fish (g/week) & $47 \pm 20$ & $74 \pm 18$ & $156 \pm 18$ & $246 \pm 20$ & 0.001 \\
\hline Vegetables $(g / d)$ & $473 \pm 37$ & $493 \pm 36$ & $465 \pm 36$ & $570 \pm 38$ & 0.21 \\
\hline Fruits $(g / d)$ & $565 \pm 37$ & $604 \pm 36$ & $565 \pm 35$ & $576 \pm 38$ & 0.13 \\
\hline Dairy $(g / d)$ & $426 \pm 38$ & $520 \pm 36$ & $501 \pm 36$ & $528 \pm 39$ & 0.23 \\
\hline Legumes $(g / d)$ & $44 \pm 5.3$ & $46 \pm 5.1$ & $46 \pm 5.1$ & $48 \pm 5.4$ & 0.96 \\
\hline
\end{tabular}

${ }^{*}$ Data are adjusted mean \pm SEM for all dietary intakes (adjusted for age and energy intake).

${ }^{* *}$ General linear model was used to compare the dietary intakes of participants across quartiles of energy-adjusted meat intake. 
Table 3. The odds ratio and 95\% CI of breast cancer across quartile categories of energy-adjusted dairy intake*

\begin{tabular}{|c|c|c|c|c|c|}
\hline & \multicolumn{5}{|c|}{$(n=274)$} \\
\hline & $Q 1$ & $Q 2$ & $Q 3$ & $Q 4$ & $P^{* *}$ \\
\hline \multicolumn{6}{|c|}{ Total meat intake } \\
\hline Model $1^{\uparrow}$ & 1 & $0.47(0.29-1.18)$ & $59(0.23-0.97)$ & $1.19(0.61-2.35)$ & 0.036 \\
\hline Model $2 \|$ & 1 & $0.75(0.30-1.89)$ & $0.95(0.81-1.27)$ & $1.46(0.29-3.13)$ & 0.11 \\
\hline Model $3^{\dagger}$ & 1 & $0.69(0.08-5.62)$ & $1.27(0.17-5.67)$ & $3.13(0.37-26.5)$ & 0.31 \\
\hline Model $4^{\ddagger}$ & 1 & $0.69(0.08-6.02)$ & $1.52(0.17-13.45)$ & $4.42(0.46-41.7)$ & 0.64 \\
\hline \multicolumn{6}{|c|}{ Red meat intake } \\
\hline Model $1^{\circ}$ & 1 & $0.67(0.37-0.71)$ & $1.62(0.14-1.72)$ & $2.38(0.02-2.36)$ & 0.006 \\
\hline Model $2 \|$ & 1 & $0.071(0.34-1.52)$ & $1.72(0.84-3.51)$ & $2.36(1.16-4.80)$ & 0.006 \\
\hline Model $3^{+}$ & 1 & $0.84(0.38-1.83)$ & $2.32(1.11-4.89)$ & $2.87(1.37-6.0)$ & 0.002 \\
\hline Model $4^{\ddagger}$ & 1 & $0.68(0.29-1.58)$ & $1.97(0.89-4.36)$ & $2.43(1.11-5.32)$ & 0.009 \\
\hline \multicolumn{6}{|c|}{ Poultry intake } \\
\hline Model 1" & 1 & $0.73(0.37-1.45)$ & $0.43(0.21-0.88)$ & $0.31(0.15-0.65)$ & 0.01 \\
\hline Model $2 \|$ & 1 & $0.72(0.36-1.46)$ & $0.47(0.22-0.97)$ & $0.38(0.17-0.83)$ & 0.06 \\
\hline Model $3^{\dagger}$ & 1 & $0.70(0.35-1.43)$ & $0.45(0.21-0.94)$ & $0.37(0.16-0.82)$ & 0.06 \\
\hline Model $4^{\ddagger}$ & 1 & $0.75(0.35-1.61)$ & $0.43(0.19-0.96)$ & $0.36(0.16-0.84)$ & 0.06 \\
\hline \multicolumn{6}{|l|}{ Fish intake } \\
\hline Model $1 "$ & 1 & $0.57(0.29-1.12)$ & $0.34(0.16-0.68)$ & $0.32(0.16-0.66)$ & 0.004 \\
\hline Model $2 \|$ & 1 & $0.55(0.28-1.09)$ & $0.34(0.16-0.69)$ & $0.31(0.15-0.65)$ & 0.005 \\
\hline Model $3^{+}$ & 1 & $0.56(0.28-1.14)$ & $0.39(0.18-0.82)$ & $0.36(0.17-0.77)$ & 0.031 \\
\hline Model $4^{\ddagger}$ & 1 & $0.59(0.29-1.21)$ & $0.37(0.17-0.80)$ & $0.37(0.17-0.82)$ & 0.024 \\
\hline \multicolumn{6}{|c|}{ Processed meat intake } \\
\hline Model $1^{\wedge}$ & 1 & $0.44(0.19-1.03)$ & $2.52(1.11-5.67)$ & $2.41(1.14-5.07)$ & 0.01 \\
\hline Model $2 \|$ & 1 & $0.16(0.06-0.43)$ & $1.25(0.52-3.04)$ & $1.81(0.76-4.29)$ & 0.001 \\
\hline Model $3^{+}$ & 1 & $0.19(0.07-0.54)$ & $1.52(0.59-3.88)$ & $2.14(0.84-5.34)$ & 0.001 \\
\hline Model $4^{\ddagger}$ & 1 & $0.26(0.08-0.77)$ & $1.77(0.65-4.77)$ & $2.15(0.80-5.74)$ & 0.001 \\
\hline
\end{tabular}

${ }^{*}$ Multivariable logistic regression models were used with adjustment of potential confounders.

** To assess the overall trends of odds ratios across quartile categories of energy-adjusted meat intake, the median of meat intake for each quartile was used as a continuous variable in logistic regression models.

" Adjusted for age (y).

Additional adjustment for age at menarche (y), age at firs pregnancy (y), number of full pregnancy, smoking (yes/no), use of oral contraceptive (yes/no) and the use of bra $(<12 \mathrm{~h} />12 \mathrm{~h})$.

${ }^{\dagger}$ Additional adjustment for body mass index $\left(\mathrm{kg} / \mathrm{m}^{2}\right)$ and life satisfaction (yes/no/partly).

"Additional adjustment for menopause status (yes/no), family history of breast cancer (yes/no), physical activity (MET-hours/week), energy intake (kcal/d), and energy density of the diet $(\mathrm{kcal} / 100 \mathrm{~g})$.

\section{Discussion}

In this study we found that despite the lack of association between dietary consumption of total meat and the risk of breast cancer, the type of meat intake could affect the odds of breast cancer independent of potentially confounding variables. Higher intake of red meat increased the odds of breast cancer and increased consumption of processed meat was accompanied with increased breast cancer risk. Our study also supported an independent protective association between higher consumption of poultry and fish meat with the risk of breast cancer.

Several case-control and prospective studies have examined the association between meat intake and breast cancer risk, however available data is not sufficient to confirm common hypothesis in this regard.

Seventeen-year follow-up of 61,433 Swedish women showed no association between total red meat intakes with breast cancer risk (17). Results from NIH-AARP Diet and Health Study cohort of 120,755 postmenopausal women provided no support for the hypothesis that intake of meat, meat cooked at high temperatures, well-done meat, or estimated intake of mutagens from meat are associated with increased risk of breast cancer (18), but seven-year follow-up of 39,268 women within the Nurses' Health Study II showed that higher red meat intake in adolescence may increase the risk of premenopausal breast cancer; the multivariateadjusted relative risk for the highest quintile of red meat intake during adolescence was 1.34 (95\% CI, 0.94-1.89; P for trend $=0.05$ ) compared with the lowest quintile, and also there was a significant linear association with every additional $100 \mathrm{~g}$ of red meat consumed per day (RR, 1.20; $95 \%$ CI, 1.00-1.43; P = 0.05). (10).

Other prospective studies also reported an elevated breast cancer risk among women with high red meat intake $(19,20)$. Moderate to strong increases in the risk of breast cancer also were observed with higher intake of total meat, red meat, beef and lamb in Uruguayan population (21). Results from one meta-analysis of case-control and cohort studies showed only a moderate association between consumption of red meat and breast cancer incidence (RR, 1.17; 95\% CI, 1.061.29) (22). Three prospective studies have investigated the association between meat intake and risk of breast cancer 
stratified by hormone receptor status of the tumour; In the Nurses' Health Study II cohort, a positive association between high intake of red meat and risk of positive estrogen receptor $(\mathrm{ER}+)$ /positive progesterone receptor (PR-) breast cancer was observed in premenopausal women (19) but in the NIH-AARP Diet and Health Study there was no association between meat intake and any subtype of breast cancer defined by hormone receptor status in postmenopausal women (18). Other prospective cohort reported that consumption of pan-fried meat increased risk of ER+/PRtumors (17).

In the current study, we found women who consumed an average of more than $156 \mathrm{~g} /$ week fish significantly were lower in the odds of breast cancer; moreover mean intake of poultry $>212 \mathrm{~g} /$ week was related to decreased the risk of breast cancer. There is limited epidemiologic evidence on the association between consumption of white meat including poultry and fish intake with breast cancer risk. A pooled analysis of 8 cohort studies from North America and Western Europe found no relation between white meat intakes with breast cancer incidence (23). In a prospective 18-year follow-up of 88,647 women, no association was observed between intakes of fish and poultry (total, without skin, with skin) with breast cancer incidence in both premenopausal and premenopausal (14). In a prospective study on postmenopausal women, there was no significant association between total fish intake and any type of fatty fish, lean fish, and boiled, fried or processed fish with incidence rate ratio of breast cancer, but additional $25 \mathrm{~g}$ daily fish intake moderately increased the risk of $\mathrm{ER}+$ breast cancer $(\mathrm{IRR}=1.14, \mathrm{CI}=1.03$ 1.26) (24). As reviewed by Stripp et al, the majority of casecontrol studies found no association between fish intake and breast cancer risk, but a limited number of these showed a significant reduction in the risk of breast cancer with increasing consumption of fish (24).

In this study, we showed a significant association between increasing trend of processed meat intake and increasing odds of breast cancer. Despite a common hypothesis in relation to consumption of processed meat and increased breast cancer risk, current data do not provide a clear association. No association was found between intake of processed meat and breast cancer risk in a prospective cohort of Swedish women. Only a borderline non-significant increased association between consumption of processed meat and the odds of breast cancer was observed among Chinese women $(\mathrm{OR}=1.44,95 \% \mathrm{CI}=0.97-2.15$; $\mathrm{P}$ for trend= 0.06) (13); a prospective cohort also showed that high intake of processed meat was associated with a modest increase in breast cancer risk (hazard ratio: 1.10; 95\% CI: 1.00, 1.20; highest compared with lowest quintile; $\mathrm{P}$ for trend $=0.07$ ) (15). In a multisite case-control study was conducted in Uruguay, consumption of processed meat more than $63 \mathrm{~g} / \mathrm{d}$ was accompanied with increased the risk of breast cancer $(\mathrm{OR}=1.53,95 \% \mathrm{CI}: 1.01-2.30)(21)$.

Several mechanisms have been proposed to explain the association between red and processed meat intake and breast cancer risk. First mechanism is attributed to high content of fat and saturated fat as established mammary carcinogens (22). Second mechanism is related to carcinogenic effects of residual amount of exogenous hormones used for growth stimulation in cattle, and heterocyclic amines and polyaromatic hydrocarbons created during the cooking of red meat $(10,25,26)$. Processed meat products, including bacon, sausage, salami and hamburger, in addition to high content of saturated and trans fats, are containing other well known carcinogenic factors such as $N$-nitroso compounds could promote mammary tumor development $(27,28)$.

To our knowledge, limited studies have examined dietary factors related to breast cancer risk in Iranian population and this study was the first investigation on the association between total meat intake and its types with breast cancer risk in Iranian women. Although small sample size and casecontrol setting were considered as weakness of the current study, but use of a validated semi-quantitative FFQ for dietary assessment, and use of several statistical models with adjustment of various known and suspected risk factors of breast cancer were the strengths of this study.

In conclusion, we found a positive association between dietary intake of red meat and processed meat products with the odds of breast cancer, as well as protective effects of fish and poultry intake with breast cancer risk in a population of Iranian women.

\section{Acknowledgments}

This study was funded by the National Nutrition and Food Technology Research Institute, Shahid Beheshti University of Medical Sciences, Tehran, Iran.

\section{References}

[1] Jemal A, Siegel R, Xu J, Ward E. Cancer statistics, 2010. CA Cancer J Clin 2010; 60(5):277-300.

[2] Ferlay J, Bray F, Pisani P, Parkin DM. GLOBOCAN 2000: Cancer Incidence, Mortality and Prevalence Worldwide IARC Cancer Base No 5 [10] Lyon, France: IARC; 2001.

[3] Bray F, McCarron P, Parkin DM. The changing global patterns of female breast cancer incidence and mortality. Breast Cancer Res 2004; 6(6):229-39.

[4] Mousavi SM, Montazeri A, Mohagheghi MA, Jarrahi AM, Harirchi I, Najafi $M$, et al. Breast cancer in Iran: an epidemiological review. Breast J 2007; 13(4):383-91.

[5] Mettlin C. Breast cancer risk factors. Contributions to planning breast cancer control. Cancer 1992; 69(7 Suppl):1904-10.

[6] Gross RE. Breast cancer: risk factors, screening, and prevention. Semin Oncol Nurs 2000; 16(3):176-84.

[7] Lee SM, Park JH, Park HJ. Breast cancer risk factors in Korean women: a literature review. Int Nurs Rev 2008; 55(3):355-9.

[8] Holmes MD, Willett WC. Does diet affect breast cancer risk? Breast Cancer Res 2006; 6(4):170-8. 
[9] Michels KB, Mohllajee AP, Roset-Bahmanyar E, Beehler GP, Moysich KB. Diet and breast cancer: a review of the prospective observational studies. Cancer 2007; 109 (12 Suppl): 2712-49.

[10] Linos E, Willett WC, Cho E, Colditz G, Frazier LA. Red meat consumption during adolescence among premenopausal women and risk of breast cancer. Cancer Epidemiol Biomarkers Prev 2008; 17(8):2146-51.

[11] Alexander DD, Morimoto LM, Mink PJ, Cushing CA. A review and meta-analysis of red and processed meat consumption and breast cancer. Nutr Res Rev 2010; 23(2):349-65.

[12] Delfino RJ, Sinha R, Smith C, West J, White E, Lin HJ, Liao SY, Gim JS, Ma HL, Butler J, Anton-Culver H. Breast cancer, heterocyclic aromatic amines from meat and Nacetyltransferase 2 genotype. Carcinogenesis 2000; 21(4):60715 .

[13] Zhang CX, Ho SC, Chen YM, Lin FY, Fu JH, Cheng SZ. Meat and egg consumption and risk of breast cancer among Chinese women. Cancer Causes Control 2009; 20(10):184553.

[14] Holmes MD, Colditz GA, Hunter DJ, Hankinson SE, Rosner B, Speizer FE, Willett WC. Meat, fish and egg intake and risk of breast cancer. Int J Cancer 2003; 104(2):221-7.

[15] Pala V, Krogh V, Berrino F, Sieri S, Grioni S, Tjønneland A, et al. Meat, eggs, dairy products, and risk of breast cancer in the European Prospective Investigation into Cancer and Nutrition (EPIC) cohort. Am J Clin Nutr 2009; 90(3):602-12.

[16] Genkinger JM, Makambi KH, Palmer JR, Rosenberg L, Adams-Campbell LL. Consumption of dairy and meat in relation to breast cancer risk in the Black Women's Health Study. Cancer Causes Control 2013.

[17] Larsson SC, Bergkvist L, Wolk A. Long-term meat intake and risk of breast cancer by oestrogen and progesterone receptor status in a cohort of Swedish women. Eur J Cancer 2009; 45(17):3042-6.

[18] Kabat GC, Cross AJ, Park Y, Schatzkin A, Hollenbeck AR, Rohan TE, Sinha R. Meat intake and meat preparation in relation to risk of postmenopausal breast cancer in the NIHAARP diet and health study. Int J Cancer 2009; 124(10):24305.

[19] Cho E, Chen WY, Hunter DJ. Red meat intake and risk of breast cancer among premenopausal women. Arch Intern Med 2006; 166: 2253-9.

[20] Taylor EF, Burley VJ, Greenwood DC, Cade JE. Meat consumption and risk of breast cancer in the UK Women's Cohort Study. Br J Cancer 2007; 96:1139-46.

[21] Aune D, De Stefani E, Ronco A, Boffetta P, Deneo-Pellegrini $\mathrm{H}$, Acosta $\mathrm{G}$, et al. Meat consumption and cancer risk: a casecontrol study in Uruguay. Asian Pac J Cancer Prev 2009; 10(3):429-36.

[22] Boyd NF, Stone J, Vogt KN, Connelly BS, Martin LJ, Minkin S. Dietary fat and breast cancer risk revisited: a meta-analysis of the published literature. Br J Cancer 2003; 89:1672-85.

[23] Missmer SA, Smith-Warner SA, Spiegelman D, Yaun SS, Adami HO, Beeson WL, et al. Meat and dairy food consumption and breast cancer: a pooled analysis of cohort studies. Int J Epidemiol 2002; 31(1):78-85.

[24] Stripp C, Overvad K, Christensen J, Thomsen BL, Olsen A, Møller S, Tjønneland A. Fish intake is positively associated with breast cancer incidence rate. J Nutr 2003; 133(11):3664-9.

[25] Fu Z, Deming SL, Fair AM, Shrubsole MJ, Wujcik DM, Shu XO, Kelley M, Zheng W. Well-done meat intake and meatderived mutagen exposures in relation to breast cancer risk: the Nashville Breast Health Study. Breast Cancer Res Treat 2011; 129(3):919-28.

[26] Lauber SN, Gooderham NJ. The cooked meat-derived mammary carcinogen 2-amino-1-methyl-6phenylimidazo[4,5-b]pyridine promotes invasive behaviour of breast cancer cells. Toxicology 2011; 279(1-3):139-45.

[27] Wu K, Sinha R, Holmes MD, Giovannucci E, Willett W, Cho E. Meat mutagens and breast cancer in postmenopausal women--a cohort analysis. Cancer Epidemiol Biomarkers Prev 2010; 19(5):1301-10.

[28] Linos E, Willett W. Meat, dairy, and breast cancer: do we have an answer? Am J Clin Nutr 2009; 90(3):455-6. 